\title{
AUDIT ON AUTISTIC SPECTRUM DISORDER (APRIL 2006 TO APRIL 2007)
}

\section{Venugopalan}

Paediatrics, Royal Berkshire Hospital, Reading, UK

Background: Autistic Spectrum disorder is a triad of impairments with 1. Impairment in social reciprocity, 2. Repititive, stereotyped, rigid patterns of unusual behavior and 3. Language abnormalities

Aim: To analyze

1. Number of children diagnosed with Autistic spectrum disorder in a year's time (April 2006 to April 2007),

2. Number of appointments needed to make ar a definite diagnosis and

3. To evaluate the co morbidities associated with ASD

Methods: Study period was from April 2006 to April 2007. All children (New \& follow up) who attended Developmental Language Clinic(DLC) were included. Numbers were obtained from

1. Outcome list from Dr Fulford (Specialist Paediatrician) and

2. Clinic letters from Paediatric Administration file, Royal Berkshire Hospital, Reading .

Results: 270 children were analysed. $72 \%$ were diagnosed to have ASD/Asperger's syndrome and $24 \%$ were diagnosed to have Specific language delay or social communication disorder.

Among the 193 with ASD/Asperger's syndrome, 139 (72\%) were diagnosed to have ASD and 55 (28\%) as Asperger's syndrome

Peak age at diagnosis was 4 years(range 2 - 11 years ). $46 \%$ were referred by GP, $28 \%$ by Speech \& Language Therapist(SALT) and $22 \%$ by Paediatricians.

$83 \%$ were diagnosed within 2 visits and 93\% were diagnosed within 3 visits

Among the comorbidities, 6\% had Sensory Modulation Disorder and 4\% had Developmental Co-ordination disorder

Conclusion: About three quarters of children referred to DLC were diagnosed to have ASD. $83 \%$ of them were diagnosed within 2 visits. All of them were referred to support groups appropriately. 